\title{
Photometric Redshift Techniques in Big-data Era
}

\author{
Yan-Xia Zhang and Yong-Heng Zhao \\ Key Laboratory of Optical Astronomy, National Astronomical Observatories, Chinese \\ Academy of Sciences, 20A Datun Road, Chaoyang District, 100012, Beijing, P.R.China \\ email:zyx@bao.ac.cn
}

\begin{abstract}
Photometric data increase with large survey projects running. The huge volume of data influences the means and methods to deal with them. As such, the techniques of photometric redshift estimation based on photometric data must be developed and improved.
\end{abstract}

Keywords. Photometric redshifts, data mining, machine learning

\section{Introduction}

Astronomy changes into a data-intensive science from a data-driven science with data deluge coming. For astronomy in the big data era see the review by Zhang \& Zhao (2015). In the big-data era, photometric redshift techniques evolve with the increase of data, original methods should combine new techniques when applied on much larger samples. Figure 1 shows that the process, techniques and tools for photometric redshift estimation, and these techniques need be interconnected to other technologies. Usually, better data is better than better algorithms, moreover better feature selection is very important. When data is large enough, the speed, usability and understanding of algorithms are taken into account. When one algorithm can not give a good result, ensemble methods are chosen.

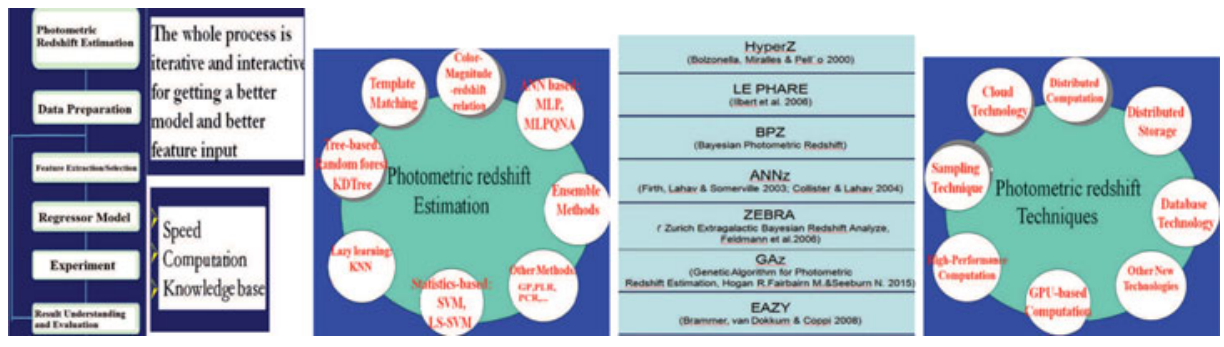

Figure 1. The first figure shows that the whole process of photometric redshift estimation; the second figure indicates the most often applied methods for photometric redshift estimation; the third figure presents the tools developed for photometric redshift estimation; the fourth figure describes that the photometric redshift techniques need be combined with other technologies to solve the similar problems faced in big-data era.

\section{Acknowledgement}

This paper is funded by National Key Basic Research Program of China 2014CB845700 and National Natural Science Foundation of China under grants No.11178021, No.11033001, NSFC-TAMU Joint Research Program No.11411120219.

\section{Reference}

Zhang, Y. \& Zhao, Y., Data Science Journal, 2015, 14(11), 1 